\title{
Mathematical Modeling, Analysis, and Optimal Control of Corruption Dynamics
}

\author{
Haileyesus Tessema Alemneh \\ Department of Mathematics, College of Natural and Computational Sciences, University of Gondar, Gondar, Ethiopia \\ Correspondence should be addressed to Haileyesus Tessema Alemneh; haila.tessema@gmail.com
}

Received 20 March 2020; Revised 16 May 2020; Accepted 8 June 2020; Published 1 August 2020

Academic Editor: Fernando Simões

Copyright (c) 2020 Haileyesus Tessema Alemneh. This is an open access article distributed under the Creative Commons Attribution License, which permits unrestricted use, distribution, and reproduction in any medium, provided the original work is properly cited.

\begin{abstract}
In this paper, a nonlinear deterministic model for the dynamics of corruption is proposed and analysed qualitatively using the stability theory of differential equations. The basic reproduction number with respect to the corruption-free equilibrium is obtained using next-generation matrix method. The conditions for local and global asymptotic stability of corruption-free and endemic equilibria are established. From the analysis using center manifold theory, the model exhibits forward bifurcation. Then, the model was extended by reformulating it as an optimal control problem, with the use of two time-dependent controls to assess the impact of corruption on human population, namely, campaigning about corruption through media and advertisement and exposing corrupted individuals to jail and giving punishment. By using Pontryagin's maximum principle, necessary conditions for the optimal control of the transmission of corruption were derived. From the numerical simulation, it was found that the integrated control strategy must be taken to fight against corruption.
\end{abstract}

\section{Introduction}

Corruption is an illegal activity carried out for private gain and benefit, by misuse of authority or power by public (government) or private (company) officeholders [1]. Different types of corruption are documented in the literature and include public corruption [2], private corruption [3], pervasive corruption, and arbitrary corruption [4]. Corruption can originate from either the demand side or the supply side [5]. In general, corruption faces a major threat to the rule of law, democracy and human rights, fairness, and social justice, hinders economic development, and brings market economies at risk for their proper and fair functioning $[2,6]$. It is a serious problem in all countries in the world mainly for developing countries [6]. Even though most countries have anticorruption policies or strategies that are being made to control corruption, it remains an epidemic in the society.

Mathematical models with optimal control analysis are an important tool in understanding the corruption transmission dynamics and in decision-making processes regarding intervention programs for corruption control. In the current research, there are only a few quantitative studies on corrup-
tion.The study by Abdulrahman [7] proposed and analysed a deterministic model for corruption in a population. They computed the basic reproduction number (BRN), corruption-free equilibrium point, and endemic equilibrium point. Numerical simulations were carried out, and they revealed that corruption can only be reduced to a manageable level but not totally removed. In [2], the authors developed and analysed a mathematical model for corruption dynamics. They determined the basic reproduction number and corruption-free and endemic equilibrium points. The study by Lemecha [8] proposed a mathematical model for corruption by considering awareness created by anticorruption and counseling in jail. The existence of unique corruption-free and endemic equilibrium points was investigated, and the basic reproduction number was computed. The study in [9] developed the differential equation-based models representing either growth or decay laws for corruption. In [10], a difference equationbased model was framed to measure the level of corruption. The author in [11] considered a game theoretic approach to deal with corruption. A corruption prevention model was formulated by Khan [12] and showed that the complete prevention of corruption is possible if the ratio between rate 
of dismissal and rate of corruption is equal to one. In [13], the authors developed an SIR model for the corruption dynamics. Furthermore, they extended the model to include optimal control with a single optimal control strategy. They concluded that the level of corruption in society can be reduced if efforts to control corruption are increased and put in place through media/punishments. Nathan and Jackob developed an epidemiological compartment model for corruption in Kenya, mainly considering those who take advantage of office holders and political office holders [14].

In this paper, an optimal control theory was used to study the effectiveness of all possible combinations of two corruption preventive measures, namely, (i) campaigning about corruption through media and advertisement and (ii) exposing the corrupted individuals to jail and giving punishment. For this, we considered a model for the transmission of corruption and incorporated two time-dependent controls. Firstly, the model has been studied with no control and the stability properties have been investigated. Secondly, we consider the control parameters to be time dependent and examine the impact of different combinations of these measures in controlling corruption. We use Pontryagin's maximum principle to derive necessary conditions for the optimal control of corruption.

The paper is organized as follows. Section 2 is devoted for the model description and the underlying assumptions. In Section 3, we carry out mathematical analysis including bifurcation and sensitivity of the model. In Section 4, the optimal control problem is presented. Section 5 is devoted for numerical simulations and discussions of the model. The conclusion is presented in Section 6 .

\section{Model Formulation and Description}

The total population $N(t)$ is divided into five compartments. Those who are susceptible to corruption are susceptible individuals $S(t)$, those who are exposed to a corrupted person but do not perform it are exposed individuals $E(t)$, those who are performing corruption are corrupted individuals $C(t)$, those who stopped doing corruption are recovered individuals $R(t)$, and those who know the badness of corruption and do not perform it permanently are honest individuals $H(t)$ at time $t \geq 0$. Assume that there is a positive recruitment $\Pi$ into the susceptible class by birth or immigration. From this class, $\kappa$ proportion will join the honest subpopulation which never involve in corruption practices irrespective of the circumstances around them. We consider a positive natural death rate $\mu$ for all humans at all time under the study. Susceptible individuals will have a contact rate with the corrupted individuals at rate $\beta$ with a corruption transmission probability $p$ per contact and moved to the exposed class. From these individuals, $\alpha$ progressed at a rate of $\delta$ to the corrupted compartment and the remaining proportion will move to the recovered compartments. The corrupted individuals get knowledge about the effect of corruption in jail and move to the recovered subpopulation at a rate of $\sigma$. From these recovered individuals, $\theta$ moved at a rate of $\varepsilon$ to susceptible compartment and the other proportion joins the honesty compartment. All the descriptions of the parameters are listed in Table 1.
With regard to the above considerations, we have the compartmental flow diagram shown in Figure 1. From the flow chart, the model will be governed by the following system of differential equations:

$$
\begin{aligned}
& \frac{d S}{d t}=\Pi+(1-\theta) \varepsilon R-p \beta S C-(k+\mu) S, \\
& \frac{d E}{d t}=p \beta S C-(\delta+\mu) E, \\
& \frac{d C}{d t}=\alpha \delta E-(\sigma+\mu) C, \\
& \frac{d R}{d t}=\sigma C+(1-\alpha) \delta E-(\varepsilon+\mu) R, \\
& \frac{d H}{d t}=\kappa S+\theta \varepsilon R-\mu H,
\end{aligned}
$$

with the initial condition

$$
\begin{gathered}
S(0)=S_{0} \geq 0, \\
E(0)=E_{0} \geq 0, \\
I(0)=I_{0} \geq 0, \\
R(0)=R_{0} \geq 0, \\
H(0)=H_{0} \geq 0 .
\end{gathered}
$$

\section{Model Analysis}

3.1. Positivity of Solutions. Since the model (1) monitors human population, it is important that all its state variables and associated parameters are positive for future time. This will be established by the following theorem:

Theorem 1. Let $\Omega=\left\{(S, E, C, R, H) \in \mathfrak{R}^{5}: S(0)>0, E(0)>\right.$ $0, C(0)>0, R(0)>0, H(0)>0\}$. Then, the solution set $(S(t)$, $E(t), C(t), R(t), H(t))$ of system (1) is positive for all $t \geq 0$.

Proof. We let $\tau=\sup \left\{t>0: S_{0}(v) \geq 0, E_{0}(v) \geq 0, C_{0}(v) \geq 0\right.$, $R_{0}(v) \geq 0, H_{0}(v) \geq 0$ for all $\left.v \in[0, t]\right\}$. Since $S_{0}(t) \geq 0, E_{0}(t) \geq$ $0, C_{0}(t) \geq 0, R_{0}(t) \geq 0$ and $H_{0}(t) \geq 0$, then $\tau>0$. If $\tau<\infty$, then automatically $S_{0}(t)$ or $E_{0}(t)$ or $C_{0}(t)$ or $R_{0}(t)$ or $H_{0}(t)$ is equal to zero at $\tau$. Taking the first equation of the model (1)

$$
\frac{d S}{d t}=\Pi+(1-\theta) \varepsilon R-p \beta S C-(k+\mu) S .
$$

Then, using the variation of constant formula the solution of equation (3) at $\tau$ is given by

$$
\begin{aligned}
S(\tau)= & S(0) \exp \left[-\int_{0}^{T}(p \beta C+(k+\mu))(s) d s\right] \\
& +\int_{0}^{t}(\Pi+(1-\theta) \varepsilon R) \\
& \cdot \exp \left[-\int_{s}^{t}(p \beta C+(k+\mu))(v) d v\right] d s>0 .
\end{aligned}
$$


TABLE 1: Description of parameters of the corruption model (1).

\begin{tabular}{lc}
\hline Parameter & Description \\
\hline$p$ & $\begin{array}{c}\text { Corruption transmission probability per contact } \\
\text { Contact rate of corrupted individual to the susceptible } \\
\text { individual }\end{array}$ \\
$\delta$ & Rate at which exposed individuals become corrupted \\
$\varepsilon$ & Rate at which corrupted individuals become recovered \\
$\Lambda$ & Rate at which recovered individuals become honest \\
$\kappa$ & Recruitment rate of susceptible humans \\
$\mu$ & $\begin{array}{c}\text { Proportion of individuals that joins the honest } \\
\text { population from susceptible population }\end{array}$ \\
$\alpha$ & Death rate of all humans \\
$\theta$ & $\begin{array}{c}\text { Proportion of individuals that joins the corrupted } \\
\text { subpopulation from the exposed compartment }\end{array}$ \\
\hline
\end{tabular}

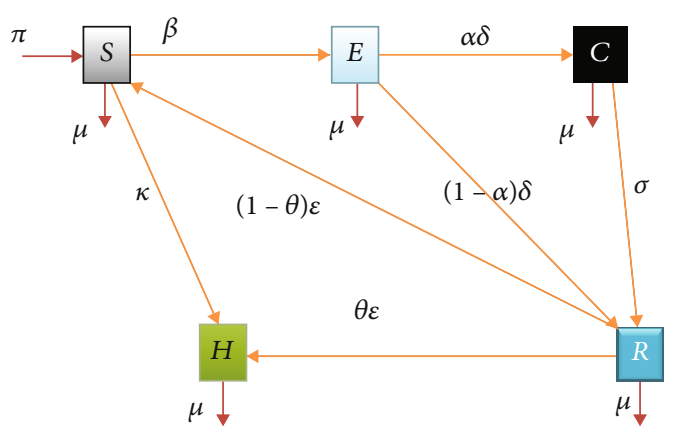

FIGURE 1: Compartmental diagram for the transmission dynamics of corruption.

Moreover, since all the variables are positive in $[0, \tau]$, hence $S(\tau)>0$. It can be shown in a similar way that $E(\tau)>0, C(\tau)$ $>0, R(\tau)>0$, and $H(\tau)>0$, which is a contradiction. Hence, $\tau=\infty$. Therefore, all the solution sets are positive for $t \geq 0$.

3.2. Invariant Region. We obtained the invariant region, in which the model solution is bounded. To do this, consider the total human population $N(t)$, where $N(t)=S(t)+E(t)+$ $C(t)+R(t)+H(t)$.

$$
\frac{d N}{d t}=\frac{d S}{d t}+\frac{d E}{d t}+\frac{d C}{d t}+\frac{d R}{d T}+\frac{d H}{d t} .
$$

Substituting all state equations from the model (1), we get

$$
\frac{d N}{d t}=\Pi-\mu N
$$
we get

Integrating equation (6) both sides and taking $t \longrightarrow \infty$,

$\Omega=\left\{(S, E, C, R, H) \in R_{+}^{5}: S(t)+E(t)+C(t)+R(t)+H(t)+\leq \frac{\Pi}{\mu}\right\}$ is positively invariant, inside which the model is considered to be epidemiologically meaningful and mathematically well posed.

3.3. Corruption-Free Equilibrium Point (CFEP). The corruption-free equilibrium point of model (1) is obtained by equating all equations of the model to zero and letting $E=0, C=0$, and $R=0$. Then, we get

$$
E_{0}=\left(\frac{\Pi}{k+\mu}, 0,0,0, \frac{k \Pi}{\mu(k+\mu)}\right) .
$$

3.4. Basic Reproduction Number. The basic reproduction number $\mathfrak{R}_{0}$ measures the expected number of secondary infections that result from one newly infected individual introduced into a susceptible population. We calculate $\mathfrak{R}_{0}$ of the model using the next-generation matrix method as described in [15]. The first step to get $\mathfrak{R}_{0}$ is rewriting the model equations starting with newly infective classes:

$$
\begin{aligned}
& \frac{d E}{d t}=p \beta S C-(\delta+\mu) E, \\
& \frac{d C}{d t}=\alpha \delta E-(\sigma+\mu) C, \\
& \frac{d R}{d t}=\sigma C+(1-\alpha) \delta E-(\varepsilon+\mu) R, \\
& \frac{d H}{d t}=\kappa S+\theta \varepsilon R-\mu H .
\end{aligned}
$$

Then, by the principle of next-generation matrix, the Jacobian, at CFEP, we obtained

$$
\begin{aligned}
F & =\left(\begin{array}{cccc}
0 & \frac{\Pi \beta p}{k+\mu} & 0 & 0 \\
0 & 0 & 0 & 0 \\
0 & 0 & 0 & 0 \\
0 & 0 & 0 & 0
\end{array}\right), \\
V & =\left(\begin{array}{cccc}
\delta+\mu & 0 & 0 & 0 \\
-\alpha \delta & \sigma+\mu & 0 & 0 \\
-(1-\alpha) \delta & -\sigma & \varepsilon+\mu & 0 \\
0 & 0 & -\theta \varepsilon & \mu
\end{array}\right) .
\end{aligned}
$$

Therefore, the basic reproduction number is the spectral radius of the next generation matrix $F V^{-1}$ and is given by

$$
\mathfrak{R}_{0}=\frac{p \beta \delta \alpha \Pi}{(\delta+\mu)(\sigma+\mu)(k+\mu)} .
$$

\subsection{Local Stability of CFEP}

Theorem 2. The CFEP is locally asymptotically stable if $\mathfrak{R}_{0}<1$ and unstable if $\mathfrak{R}_{0}>1$. 
Proof. To prove this theorem, let us first find the Jacobian matrix of system (1):

$J=\left(\begin{array}{ccccc}-\beta p C-(\kappa+\mu) & 0 & -p \beta S & (1-\theta) \varepsilon & 0 \\ \beta p C & -\delta-\mu & p \beta S & 0 & 0 \\ 0 & \alpha \delta & -\mu-\sigma & 0 & 0 \\ 0 & (1-\alpha) \delta & \sigma & -(\varepsilon+\mu) & 0 \\ \kappa & 0 & 0 & \theta \varepsilon & -\mu\end{array}\right)$.

Evaluating equation (12) at the corruption-free equilibrium $E_{0}=(\Pi /(k+\mu), 0,0,0, \Pi / \mu(k+\mu))$, we got

$$
J=\left(\begin{array}{ccccc}
-(\kappa+\mu) & 0 & -\frac{p \beta \Pi}{\kappa+\mu} & (1-\theta) \varepsilon & 0 \\
0 & -\delta-\mu & \frac{p \beta \Pi}{\kappa+\mu} & 0 & 0 \\
0 & \alpha \delta & -\mu+\sigma & 0 & 0 \\
0 & (1-\alpha) \delta & \sigma & -(\varepsilon+\mu) & 0 \\
\kappa & 0 & 0 & \theta \varepsilon & -\mu
\end{array}\right) .
$$

From the Jacobian matrix, a characteristic polynomial was obtained as

$$
(-\lambda-(\kappa+\mu))(-\lambda-\mu)(-\lambda-(\varepsilon+\mu))\left[\lambda^{2}+\psi_{1} \lambda+\psi_{2}\right]=0,
$$

where

$$
\begin{gathered}
\psi_{1}=\sigma+2 \mu+\delta \\
\psi_{2}=\frac{-\Pi \alpha \beta \delta p+(\delta+\mu)(\kappa+\mu)(\sigma+\mu)}{\kappa+\mu} .
\end{gathered}
$$

From equation (14), we have

$$
\begin{aligned}
-\lambda-(\kappa+\mu) & \Rightarrow \lambda_{1}=-(\kappa+\mu)<0, \\
-\lambda-\mu & \Rightarrow \lambda_{2}=-\mu<0, \\
-\lambda-(\varepsilon+\mu) & \Rightarrow \lambda_{3}=-(\varepsilon+\mu)<0 .
\end{aligned}
$$

From the last expression of equation (14), we have

$$
\lambda^{2}+\psi_{1} \lambda+\psi_{2}=0
$$

The Routh-Hurwitz criteria was applied, and equation (17) has strictly negative real root iff $\psi_{1}>0$ and $\psi_{2}>0$. Clearly, we see that $\psi_{1}>0$ because it is the sum of positive parameters and also

$$
\begin{aligned}
\psi_{2} & =\frac{-\Pi \alpha \beta \delta p+(\delta+\mu)(\kappa+\mu)(\sigma+\mu)}{\kappa+\mu} \\
& =(\delta+\mu)(\sigma+\mu)\left(1-\mathfrak{R}_{0}\right)>0 .
\end{aligned}
$$

Hence, the CFEP is locally asymptotically stable if $\Re_{0}<1$.

This result implies that corruption can be eliminated if the initial size of the corrupted individuals is in the basin of attraction of the CFEP. On the other hand, to ensure that corruption elimination is independent of the initial population size of the corrupted population, it is necessary to show that the CFEP is globally stable.

\subsection{Global Stability of CFEP}

Theorem 3. The equilibrium point $E_{0}$ of the model (1) is globally asymptotically stable if $\mathfrak{R}_{0}<1$.

Proof. Consider the following Lyapunov function

$$
V=\chi_{1} E+\chi_{2} C
$$

Differentiating equation (19) with respect to $t$ gives

$$
\frac{d V}{d t}=\chi_{1} \frac{d E}{d t}+\chi_{2} \frac{d C}{d t}
$$

Substituting $d E / d t$ and $d C / d t$ from the model (1), we get

$$
\begin{aligned}
\frac{d V}{d t} & =\chi_{1}[(p \beta \delta C)-(\delta+\mu) E]+\chi_{2}[\alpha \delta E-(\sigma+\mu) C] \\
& =\chi_{1} p \beta \delta C-\chi_{2}(\sigma+\mu) C-\chi_{1}(\delta+\mu) E+\chi_{2} \alpha \delta E .
\end{aligned}
$$

Here, take $\chi_{1}=(\alpha \delta /(\delta+\mu)) \chi_{2}$, then we have

$$
\begin{aligned}
\frac{d V}{d t} & =\frac{\alpha \delta}{\delta+\mu} \chi_{2} \beta \delta C-\chi_{2}(\sigma+\mu) C \\
& \leq\left[\frac{p \alpha \beta \delta \Pi}{(\delta+\mu)(k+\mu)}-(\sigma+\mu)\right] \chi_{2}{ }^{C} .
\end{aligned}
$$

Taking $\chi_{2}=1$ and substituting $\mathfrak{R}_{0}$, we get

$$
\frac{d V}{d t} \leq(\sigma+\mu)\left(\Re_{0}-1\right) C
$$

for $C \leq C^{0}=\pi /(k+\mu)$ and $d V / d t \leq 0$ for $\mathfrak{R}_{0}<1$ and $d V /$ $d t=0$ if and only if $C=0$. This implies that the only trajectory of the system (1) on which $d V / d t \leq 0$ is $E^{0}$. Therefore, by LaSalle's invariance principle, $E^{0}$ is globally asymptotically stable in $\Omega$. This supports the forward bifurcation of Section 3.9.

3.7. The Endemic Equilibrium Point. The endemic equilibrium point denoted by $E^{*}=\left(S^{*}, E^{*}, C^{*}, R^{*}, H^{*}\right) . E^{*}$ is the steady state solution where corruption persists in the 
population. It can be obtained by equating each equation in (1) to zero:

$$
\frac{d S}{d t}=\frac{d E}{d t}=\frac{d C}{d t}=\frac{d R}{d t}=\frac{d H}{d t}=0 .
$$

Then, we obtain

$$
\begin{aligned}
S^{*} & =\frac{(\delta+\mu)(\sigma+\mu)}{\alpha \beta \delta p}, \\
E^{*} & =\frac{(\sigma+\mu)(\varepsilon+\mu)\left[1-\mathfrak{R}_{0}\right]}{\alpha \delta p \beta \phi(\delta+\mu)(\sigma+\mu)(\kappa+\mu)}, \\
C^{*} & =\frac{(\varepsilon+\mu)\left[1-\mathfrak{R}_{0}\right]}{p \beta \phi(\delta+\mu)(\sigma+\mu)(\kappa+\mu)}, \\
R^{*} & =\frac{[\mu(\alpha-1)-\sigma]\left[\mathfrak{R}_{0}-1\right]}{\alpha p \beta \phi(\delta+\mu)(\sigma+\mu)(\kappa+\mu)}, \\
H^{*} & =\frac{\kappa(\delta+\mu)(\sigma+\mu)}{\alpha \delta p \beta \mu}+\frac{\varepsilon \theta[\mu(\alpha-1)-\sigma]\left[\mathfrak{R}_{0}-1\right]}{\alpha p \beta \phi \mu(\delta+\mu)(\sigma+\mu)(\kappa+\mu)},
\end{aligned}
$$

where

$$
\phi=\alpha \delta \mu(\theta-1)-(\varepsilon+\mu)(\delta+\mu)(\sigma+\mu)-\delta(1+\theta)(\sigma+\mu) .
$$

\subsection{Local Stability of Endemic Equilibrium}

Theorem 4. The endemic equilibrium $E^{*}$ of system (1) is locally asymptotically stable in $\Omega$ if $\Re_{0}>1$, otherwise unstable.

Proof. Let us first obtain the Jacobian matrix of system (1):

$$
J=\left(\begin{array}{ccccc}
-\beta p C-(\kappa+\mu) & 0 & -p \beta S & (1-\theta) \varepsilon & 0 \\
\beta p C & -(\delta+\mu) & p \beta S & 0 & 0 \\
0 & \alpha \delta & -(\mu+\sigma) & 0 & 0 \\
0 & (1-\alpha) \delta & \sigma & -(\varepsilon+\mu) & 0 \\
\kappa & 0 & 0 & \theta \varepsilon & -\mu
\end{array}\right) .
$$

The characteristic polynomial of equation (27) is given by

$$
\lambda^{5}+\psi_{1} \lambda^{4}+\psi_{2} \lambda^{3}+\psi_{3} \lambda^{2}+\psi_{2} \lambda+\psi_{4}=0
$$

where

$$
\begin{aligned}
\psi_{1}= & \frac{(\sigma+\mu)(\varepsilon+\mu)\left[\Re_{0}-1\right]}{\alpha \delta \phi}+\delta+\epsilon+\kappa+5 \mu+\sigma, \\
\psi_{2}= & -(\delta+\mu)(\sigma+\mu)+(\delta+\varepsilon+\sigma+4 \mu)\left[\frac{(\varepsilon+\mu)\left[\Re_{0}-1\right]}{\phi}+\delta\right] \\
& +(4 \mu+\sigma)(\epsilon+\kappa)+\varepsilon \kappa+\mu(10 \mu+4 \sigma),
\end{aligned}
$$

$$
\begin{aligned}
\psi_{3}= & -(\delta+\mu)(\sigma+\mu)(\varepsilon+\kappa+3 \mu)+\frac{(\varepsilon+\mu)\left[\mathfrak{R}_{0}-1\right]}{\phi}\{\delta(1 \\
& -\theta+\sigma+3 \mu)+\varepsilon+3 \mu(2 \mu+\sigma)-\alpha \delta(\theta-1)\} \\
& +(\sigma+3 \mu)[\delta \kappa+\varepsilon \kappa+\delta \varepsilon]+(2 \mu+\sigma)[3 \varepsilon \mu-3 \kappa \mu-6 \delta \mu] \\
& +\delta \varepsilon \kappa+2 \mu^{2}(5 \mu+\sigma), \\
\psi_{4}= & 2 \beta p C[\alpha \delta \theta(\sigma+\mu)+\alpha \delta(\mu+\sigma)(\theta+1)+(\sigma+\mu) \delta(\varepsilon+1)] \\
& +\beta p C[\delta \mu(3 \mu+2 \sigma-2 \theta)+\delta \sigma \theta+\varepsilon \mu(3 \mu+2 \sigma) \\
& \left.+\mu^{2}(4 \mu+3 \sigma)\right]+2(\mu+\sigma) \delta \varepsilon \kappa+(3 \mu+2 \sigma)[\delta \varepsilon \mu+\delta \kappa p \\
& +\varepsilon \kappa p]-\mu^{2}(4 \mu+3 \sigma)[\varepsilon+\kappa+\delta]+\mu^{3}(5 \mu+4 \sigma) \\
& -(\delta+\mu)(\sigma+\mu)\left(\varepsilon \kappa+2 \varepsilon \mu+2 \kappa \mu+3 \mu^{2}\right), \\
\psi_{5}= & -\mu(\varepsilon+\mu)[\Re-1][(\mu+2 \sigma) \alpha \delta(\theta-1) \\
& -(\sigma+\mu)[\beta \delta(\varepsilon+\mu-\theta)+\beta \mu(\varepsilon+\mu+\delta)] \\
& -(\kappa+\mu)(\delta+\mu)(\sigma+\mu) \mu\left[\frac{\varepsilon}{\beta} \beta \mu+\beta \kappa \mu\right] \\
& +(\sigma+\mu) \mu[\varepsilon \kappa(\beta+\mu)+\mu \delta(\kappa+\varepsilon) \\
& \left.+\mu^{2}(\delta+\varepsilon+\kappa+\mu)\right],
\end{aligned}
$$

Using the Routh-Hurwitz criterion, all roots of characteristic polynomial have negative real parts if and only if $\psi_{1}>0, \psi_{2}>0, \psi_{3}>0, \psi_{4}>0, \psi_{5}>0, \psi_{1} \psi_{2} \psi_{3}>\psi_{3}^{2}+\psi_{1}^{2} \psi_{4}$ and $\left(\psi_{1} \psi_{4}-\psi_{5}\right)\left(\psi_{1} \psi_{2} \psi_{3}-\psi_{3}^{2}+\psi_{1}^{2} \psi_{4}\right)>\psi_{5}\left(\psi_{1} \psi_{2}-\psi_{3}\right)^{2}+$ $\psi_{1} \psi_{5}^{2}$ for $\Re_{0}>1$. Hence, the endemic equilibrium $E^{*}$ is locally asymptotically stable.

3.9. Bifurcation Analysis. In order to determine the direction of the bifurcation and to prove the stability of the endemic equilibrium point, we make use of the bifurcation theory, which is based on the center manifold theory [16] (as described by Theorem 6 of Castillo-Chavez and Song [16]).

Theorem 5. The model in the system (1) exhibits forward bifurcation at $\boldsymbol{R}_{0}=1$ and a unique endemic equilibrium exists.

Proof. To conduct this analysis, we assume that $S=z_{1}$, $E=z_{2}, C=z_{3}, R=z_{4}, H=z_{5}$ and from $\Re_{0}=1$, we have

$$
\beta=\beta *=\frac{(\delta+\mu)(\sigma+\mu)(k+\mu)}{p \delta \alpha \Pi} .
$$

The new the model in equation (1) is given by

$$
\begin{aligned}
\frac{d z_{1}}{d t} & =\Pi+(1-\theta) \varepsilon z_{4}-p \beta z_{1} z_{3}-(k+\mu) z_{1}, \\
\frac{d z_{2}}{d t} & =p \beta z_{1} z_{3}-(\delta+\mu) z_{2}, \\
\frac{d z_{3}}{d t} & =\alpha \delta z_{2}-(1-\mu) z_{3}, \\
\frac{d z_{4}}{d t} & =\sigma z_{3}+(1-\alpha) \delta z_{2}-(\varepsilon+\mu) z_{4}, \\
\frac{d z_{5}}{d t} & =\kappa z_{1}+\theta \varepsilon z_{4}-\mu z_{5} .
\end{aligned}
$$


The Jacobian of the model system (31) around the corruption-free equilibrium point $E_{0}=\left(z_{1}=\Pi /(\kappa+\mu), 0\right.$, $\left.0,0, z_{5}=\kappa \Pi / \mu(\kappa+\mu)\right)$ evaluated at $\mathfrak{R}_{0}=1$ is

$$
J=\left(\begin{array}{ccccc}
-(\kappa+\mu) & 0 & -\frac{p \beta \Pi}{\kappa+\mu} & (1-\theta) \varepsilon & 0 \\
0 & -\delta-\mu & \frac{p \beta \Pi}{\kappa+\mu} & 0 & 0 \\
0 & \alpha \delta & -\mu+\sigma & 0 & 0 \\
0 & (1-\alpha) \delta & \sigma & -(\varepsilon+\mu) & 0 \\
\kappa & 0 & 0 & \theta \varepsilon & -\mu
\end{array}\right) .
$$

The right eigenvector, $w=\left(w_{1}, w_{2}, w_{3}, w_{4}, w_{5}\right)^{T}$, associated with this simple zero eigenvalue can be obtained from $J w=0$.

$$
\begin{aligned}
w_{1}= & {\left[\frac{(1-\theta) \varepsilon[(1-\alpha) \delta(\alpha+\mu)+\sigma \delta \alpha]}{(\sigma+\mu)(\varepsilon+\mu)(\kappa+\mu)}-\frac{\delta+\mu}{\kappa+\mu}\right] w_{2}, } \\
w_{2}= & w_{2}>0, \\
w_{3}= & \frac{\alpha \delta}{\mu+\sigma} w_{2}, \\
w_{4}= & \frac{(1-\alpha) \varepsilon(\sigma+\mu)+\sigma \delta \alpha}{(\mu+\sigma)(\varepsilon+\mu)} w_{2}, \\
w_{5}= & \frac{\kappa}{\mu}\left[\frac{(1-\theta) \varepsilon[(1-\alpha) \delta(\sigma+\mu)+\sigma \delta \alpha]}{(\sigma+\mu)(\varepsilon+\mu)(\kappa+\mu)}-\frac{\delta+\mu}{\kappa+\mu}\right] w_{2} \\
& +\frac{\theta \varepsilon[(1-\alpha) \delta(\sigma+\mu)+\sigma \delta \alpha]}{\mu(\mu+\sigma)(\varepsilon+\mu)} w_{2} .
\end{aligned}
$$

The left eigenvector, $v=\left(v_{1}, v_{2}, v_{3}, v_{4}, v_{5}\right)$, associated with this simple zero eigenvalue can be obtained from $v J=0$ and given by

$$
\begin{aligned}
& v_{1}=v_{4}=v_{5}=0, \\
& v_{2}=v_{2}>0, \\
& v_{3}=\frac{\delta+\mu}{\alpha \delta} v_{2} .
\end{aligned}
$$

Since the first, fourth, and fifth components of $v$ are zero, we do not need the derivatives of $f_{1}, f_{4}$, and $f_{5}$. From the derivatives of $f_{2}$ and $f_{3}$, the only ones that are nonzero are the following:

$$
\begin{aligned}
\frac{\partial^{2} f_{2}}{\partial z_{2} \partial z_{3}} & =\frac{\partial^{2} f_{2}}{\partial z_{3} \partial z_{2}}=p \beta, \\
\frac{\partial^{2} f_{2}}{\partial z_{2} \partial \beta} & =p z_{1}^{*},
\end{aligned}
$$

and all the other partial derivatives are zero. The direction of the bifurcation at $\mathfrak{R}_{0}=1$ is determined by the signs of the bifurcation coefficients $a$ and $b$, obtained from the above partial derivatives, given, respectively, by
Table 2: Sensitivity index table.

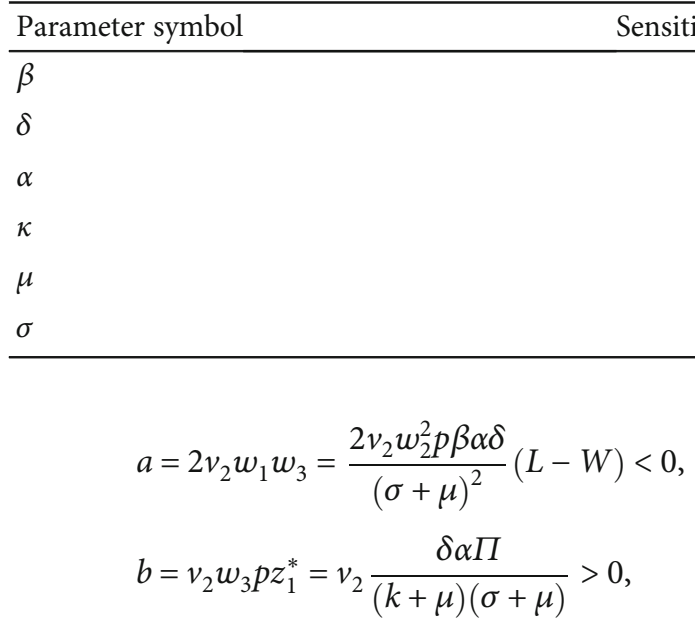

where

$$
\begin{aligned}
L & =\frac{\varepsilon \delta[(1-\theta)(\sigma+\mu)+\theta \alpha(2 \sigma+\mu)]}{(\varepsilon+\mu)(\kappa+\mu)}, \\
W & =\frac{\varepsilon \alpha \delta \mu+(\varepsilon+\mu)(\delta+\mu)^{2}}{(\varepsilon+\mu)(\kappa+\mu)} .
\end{aligned}
$$

Since the coefficient $b$ is positive and the sign of the coefficient $a$ is negative, the model undergoes forward bifurcation, which implies that $\mathfrak{R}_{0}<1$ is a necessary and sufficient condition for corruption elimination.

3.10. Sensitivity Analysis. In order to see the relative effect of each parameter to the corruption transmission, we performed sensitivity analysis. To go through, we followed the approach defined by [17] as done in [18, 19] which used the definition of normalized forward sensitivity index defined as a variable, $\mathfrak{R}_{0}$, that depends differentiably on a parameter, $\ell$, defined as

$$
\Lambda_{\ell}^{\mathfrak{R}_{0}}=\frac{\partial \mathfrak{R}_{0}}{\partial \ell} \times \frac{\ell}{\mathfrak{R}_{0}},
$$

for $\ell$ represents all the basic parameters and $\mathfrak{R}_{0}=\alpha p \beta \delta \Pi$ $/(\delta+\mu)(\sigma+\mu)(\kappa+\mu)$.

$$
\begin{aligned}
& \Lambda_{\kappa}^{\mathfrak{R}_{0}}=\frac{\partial \mathfrak{R}_{0}}{\partial \beta} \times \frac{\beta}{\mathfrak{R}_{0}}=1 \geq 0, \\
& \Lambda_{\mathcal{\kappa}}^{\mathfrak{R}_{0}}=\frac{\partial \mathfrak{R}_{0}}{\partial \kappa} \times \frac{\beta}{\mathfrak{R}_{0}}=-\frac{\kappa}{\kappa+\mu} \leq 0, \\
& \Lambda_{\sigma}^{\Re_{0}}=\frac{\partial \mathfrak{R}_{0}}{\partial \sigma} \times \frac{\beta}{\mathfrak{R}_{0}}=-\frac{\sigma}{\sigma+\mu} \leq 0 .
\end{aligned}
$$

And we do this in a similar fashion for the remaining parameters.

3.11. Interpretation of Sensitivity Indices. The sensitivity indices of parameters are found in Table 2. Parameters that have positive indices $(\beta, \alpha$, and $\delta)$ have great impact on expanding the corruption in the community when their 
values increase. Parameters with negative indices $(\kappa, \sigma$, and $\mu)$ minimize the burden of corruption in the community as their values increase.

Therefore, the model sensitivity analysis demonstrated that policy makers are supposed to decrease positive index parameters and increase negative index parameters to combat corruption in a population. From this result, to identify the optimal strategy to fight against corruption, an optimal control model analysis is considered in the next section.

\section{Extension of the Model into Optimal Control}

The optimal level that would be needed to control corruption is minimizing the number of exposed and corrupted population. An optimal control model was developed by including the following two controls:

(i) $u_{1}$ : campaigning about corruption through media and advertisement

(ii) $u_{2}$ : exposing the corrupted individuals to jail and giving punishment

Adding the two controls on the model (1), the optimal control model is given by

$$
\begin{aligned}
& \frac{d S}{d t}=\Pi+(1-\theta) \varepsilon R-\left(1-u_{2}\right) p \beta S C-\left(k+\mu+u_{1}\right) S, \\
& \frac{d E}{d t}=\left(1-u_{2}\right) p \beta S C-\left(\delta+\mu+u_{1}\right) E, \\
& \frac{d C}{d t}=\left(1-u_{1}\right) \alpha \delta E-\left(\sigma+\mu+u_{2}\right) C, \\
& \frac{d R}{d t}=\left(\sigma+u_{2}\right) C+(1-\alpha)\left(\delta+u_{2}\right) E-(\varepsilon+\mu) R, \\
& \frac{d H}{d t}=\left(k-u_{1}\right) S+\theta \varepsilon R-\mu H .
\end{aligned}
$$

The control variables $u_{1}$ and $u_{2}$ minimize the optimal control model (40) subject to the objective functional defined as

$$
J=\int_{0}^{t_{f}}\left[a_{1} E+a_{2} I+\frac{1}{2}\left(w_{1} u_{1}^{2}+w_{2} u_{2}^{2}\right)\right] d t
$$

where $t_{f}$ is the final time, $a_{1}$ and $a_{2}$ are weight constants of the exposed and corrupted population, respectively, while $w_{1}$ and $w_{2}$ are weight coefficients for each individual control measure. We choose a nonlinear cost on the controls based on the assumption that the cost takes nonlinear form [20]. Optimal control function $\left(u_{1}^{*}, u_{2}^{*}\right)$ needs to be found such that

$$
J\left(u_{1}^{*}, u_{2}^{*}\right)=\min \left\{J\left(u_{1}, u_{2}\right) \mid\left(u_{1}, u_{2}\right) \in U\right\}
$$

where $U=\left\{\left(u_{1}, u_{2}\right) \mid u_{i}(t)\right.$ is lebesgue measurable on $\left[0, t_{f}\right]$, $\left.0 \leq u_{i}(t) \leq 1, i=1,2\right\}$ is the closed set.
4.1. The Hamiltonian and Optimality System. The optimal control must satisfy the necessary conditions that are formulated by Pontryagin's maximum principle ([21]). This principle converts the system of equations (40) and (41) into a problem of minimizing point-wise a Hamiltonian $(M)$, with respect to $u_{1}(t)$ and $u_{2}(t)$ as

$$
\begin{aligned}
M= & a_{1} E+a_{2} C+\frac{1}{2}\left(w_{1} u_{1}^{2}+w_{2} u_{2}^{2}+w_{3} u_{3}^{2}\right) \\
& +\lambda_{1}\left[\Pi+(1-\theta) \varepsilon R-\left(1-u_{2}\right) p \beta S C-\left(k+\mu+u_{1}\right) S\right] \\
& +\lambda_{2}\left[\left(1 \mid-u_{2}\right) p \beta S C-\left(\delta+\mu+u_{1}\right) E\right]+\lambda_{3}\left[\left(1-u_{1}\right) \alpha \delta E\right. \\
& \left.-\left(\sigma+\mu+u_{2}\right) C\right]+\lambda_{4}\left[\left(\sigma+u_{2}\right) C+(1-\alpha)\left(\delta+u_{2}\right) E\right. \\
& -(\varepsilon+\mu) R]+\lambda_{5}\left[\left(k+u_{1}\right) S+\theta \varepsilon R-\mu H\right],
\end{aligned}
$$

where $\lambda_{i}, i=1, \cdots, 5$ are the adjoint variable functions to be determined. Because of the convexity of the integrand of $J$ with respect to $u_{1}$ and $u_{2}$, a priori boundaries of the state solutions and the Lipschitz property of the state system relating to the state variables, the existence of the optimal control was proved using Corollary 4.1 in the work of Fleming and Rishel [22].

Theorem 6. Suppose we have optimal controls $u_{1}^{*}, u_{2}^{*}$ and $S, E, C, R, H$ solutions of the respective state system that minimizes $J$ over $U$, there exist adjoint variables, $\lambda_{1}, \cdots$, $\lambda_{5}$ such that

$$
\begin{aligned}
\frac{d \lambda_{1}}{d t}= & \lambda_{1}\left(\left(1-u_{2}\right) p \beta C+\kappa+\mu+u_{1}\right) \\
& -\lambda_{2}\left(1-u_{2}\right) p \beta C-\lambda_{5}\left(\kappa+u_{1}\right), \\
\frac{d \lambda_{2}}{d t}= & -a_{1}+\lambda_{2}\left(\delta+\mu+u_{1}\right)-\lambda_{3} \alpha \delta\left(1-u_{1}\right) \\
& -\lambda_{4}(1-\alpha)\left(\delta+u_{1}\right), \\
\frac{d \lambda_{3}}{d t}= & -a_{2}+\lambda_{1}\left(1-u_{2}\right) p \beta S-\lambda_{2}\left(1-u_{2}\right) p \beta S \\
& +\lambda_{3}\left(\sigma+\mu+u_{2}\right)-\lambda_{4}\left(\sigma+u_{2}\right), \\
\frac{d \lambda_{4}}{d t}= & \lambda_{1} \in(1-\theta)+\lambda_{4}(\epsilon+\mu)-\lambda_{5} \theta \epsilon, \\
\frac{d \lambda_{5}}{d t}= & \mu \lambda_{5} .
\end{aligned}
$$

With transversality conditions, $\quad \lambda_{i}\left(t_{f}\right)=0, i=1, \cdots, 5$. Furthermore, we obtained the control set $\left(u_{1}^{*}, u_{2}^{*}\right)$ characterized by

$$
\begin{aligned}
& u_{1}^{*}=\max \left\{0, \min \left(1, \frac{\left(\lambda_{1}-\lambda_{5}\right) S+\left[\lambda_{2}+\alpha \delta \lambda_{3}-(1-\alpha) \lambda_{4}\right] E}{w_{1}}\right)\right\}, \\
& u_{2}^{*}=\max \left\{0, \min \left(1, \frac{p \beta C S\left(\lambda_{2}-\lambda_{1}\right)+\left(\lambda_{4}-\lambda_{3}\right) C}{w_{2}}\right)\right\} .
\end{aligned}
$$


Proof. The adjoint equation and transversality conditions are standard results obtained from Pontryagin's maximum principle [21]. Differentiate the Hamiltonian with respect to states $S, E, C, R$, and $H$, respectively, then we got the adjoint equations as

$$
\begin{aligned}
\frac{d \lambda_{1}}{d t}= & -\frac{\partial M}{\partial S}=\lambda_{1}\left(\left(1-u_{2}\right) p \beta C+\kappa+\mu+u_{1}\right) \\
& -\lambda_{2}\left(1-u_{2}\right) p \beta C-\lambda_{5}\left(\kappa+u_{1}\right), \\
\frac{d \lambda_{2}}{d t}-\frac{\partial M}{\partial E}= & -a_{1}+\lambda_{2}\left(\delta+\mu+u_{1}\right)-\lambda_{3} \alpha \delta\left(1-u_{1}\right) \\
& -\lambda_{4}(1-\alpha)\left(\delta+u_{1}\right), \\
\frac{d \lambda_{3}}{d t}-\frac{\partial M}{\partial C}= & -a_{2}+\lambda_{1}\left(1-u_{2}\right) p \beta S-\lambda_{2}\left(1-u_{2}\right) p \beta S \\
& +\lambda_{3}\left(\sigma+\mu+u_{2}\right)-\lambda_{4}\left(\sigma+u_{2}\right), \\
\frac{d \lambda_{4}}{d t}-\frac{\partial M}{\partial R}= & -\lambda_{1} \in(1-\theta)+\lambda_{4}(\epsilon+\mu)-\lambda_{5} \theta \epsilon, \\
& \frac{d \lambda_{5}}{d t}-\frac{\partial M}{\partial H}=\mu \lambda_{5} .
\end{aligned}
$$

With transversality conditions, $\lambda i(t f)=0, i=1, \cdots, 5$. Differentiate the Hamiltonian equation with respect to $u_{1}$ and $u_{2}$ and obtain the characterization of optimal controls $u_{1}^{*}, u_{2}^{*}$ from $\partial M / \partial u_{1}=0, \partial M / \partial u_{2}=0$. Thus, we got the characteristic equation in standard control arguments form involving the bounds on the controls as follows:

$$
\begin{aligned}
& u_{1}^{*}= \begin{cases}\psi_{1}, & \text { if } 0<\psi_{1}<1, \\
0, & \text { if } \psi_{1} \leq 0 \\
1, & \text { if } \psi_{1} \geq 1,\end{cases} \\
& u_{2}^{*}= \begin{cases}\psi_{2}, & \text { if } 0<\psi_{2}<1, \\
0, & \text { if } \psi_{2} \leq 0 \\
1, & \text { if } \psi_{2} \geq 1\end{cases}
\end{aligned}
$$

In compacted notation:

$$
\begin{aligned}
& u_{1}^{*}=\max \{0, \min (1, \psi 1)\}, \\
& u_{2}^{*}=\max \{0, \min (1, \psi 2)\},
\end{aligned}
$$

where

$$
\begin{aligned}
& \psi_{1}=\frac{\left(\lambda_{1}-\lambda_{5}\right) S+\left[\lambda_{2}+\alpha \delta \lambda_{3}-(1-\alpha) \lambda_{4}\right] E}{w_{1}}, \\
& \psi_{2}=\frac{p \beta C S\left(\lambda_{2}-\lambda_{1}\right)+\left(\lambda_{4}-\lambda_{3}\right) C}{w_{2}} .
\end{aligned}
$$

The optimality system is formed from the optimal control system (the state system) and the adjoint variable
TABLE 3: Values of parameter used in the numerical simulation.

\begin{tabular}{lcc}
\hline Parameter & Value & Source \\
\hline$\Pi$ & 85 & Assumed \\
$p$ & 0.036 & {$[7,8]$} \\
$\beta$ & 0.0234 & {$[7,8]$} \\
$\delta$ & 0.2 & Assumed \\
$\sigma$ & 0.007 & Assumed \\
$\varepsilon$ & 0.35 & Assumed \\
$\kappa$ & 0.03 & {$[8]$} \\
$\mu$ & 0.0160 & {$[8]$} \\
$\alpha$ & 0.3 & {$[8]$} \\
$\theta$ & 0.1 & {$[8]$} \\
\hline
\end{tabular}

system by incorporating the characterized control set under initial and transversal condition:

$\left\{\begin{array}{l}\frac{d S}{d t}=\prod+(1-\theta) \varepsilon R-\left(1-u_{2}^{*}\right) p \beta S C-\left(k+\mu+u_{1}^{*}\right) S, \\ \frac{d E}{d t}=\left(1-u_{2}^{*}\right) p \beta S C-\left(\delta+\mu+u_{1}^{*}\right) E, \\ \frac{d C}{d t}=\left(1-u_{1}^{*}\right) \alpha \delta E-\left(\alpha+\mu+u_{2}^{*}\right) C, \\ \frac{d R}{d t}=\left(\sigma+u_{2}^{*}\right) C+(1-\alpha)\left(\delta+u_{2}^{*}\right) E-(\varepsilon+\mu) R, \\ \frac{d H}{d t}=\left(k+u_{1}^{*}\right) S+\theta \varepsilon R-\mu H, \\ \frac{d \lambda_{1}}{d t}=\lambda_{1}\left(\left(1-u_{2}^{*}\right) p \beta C+k+\mu+u_{1}^{*}\right)-\lambda_{2}\left(1-u_{2}^{*}\right) p \beta C-\lambda_{5}\left(k+u_{1}^{*}\right), \\ \frac{d \lambda_{2}}{d t}=-a_{1}+\lambda_{2}\left(\delta+\mu+u_{1}^{*}\right)-\lambda_{3} \alpha \delta\left(1-u_{1}^{*}\right)-\lambda_{4}(1-\alpha)\left(\delta+u_{1}^{*}\right), \\ \frac{d \lambda_{3}}{d t}=-a_{2}+\lambda_{1}\left(1-u_{2}^{*}\right) p \beta S-\lambda_{2}\left(1-u_{2}^{*}\right) p \beta S+\lambda_{3}\left(\sigma+\mu+u_{2}^{*}\right)-\lambda_{4}\left(\sigma+u_{2}^{*}\right), \\ \frac{d \lambda_{4}}{d t}=-\lambda_{1} \in(1-\theta)+\lambda_{4}(\epsilon+\mu)-\lambda_{5} \theta \varepsilon, \\ \frac{d \lambda_{5}}{d t}=\mu \lambda_{5}, \\ u_{1}^{*}=\max \left\{0, \min \left(1, \frac{\left(\lambda_{1}-\lambda_{5}\right) S+\left[\lambda_{2}+\alpha \delta \lambda_{3}-(1-\alpha) \lambda_{4}\right] E}{w_{1}}\right)\right\}, \\ u_{2}^{*}=\max \left\{0, \min \left(1, \frac{p \beta C S\left(\lambda_{2}-\lambda_{1}\right)+\left(\lambda_{4}-\lambda_{3}\right) C}{w_{2}}\right)\right\}, \\ \lambda_{i}\left(t_{f}\right)=0 \\ i=1, \cdots, 5 \\ S(0)=S_{0}, \\ E(0)=E_{0}, \\ C(0)=C_{0}, \\ R(0)=R_{0}, \\ H(0)=H_{0} .\end{array}\right.$

\section{Numerical Simulations and Discussions}

In this section, numerical simulations are performed on the model (1). Maple 18 software is used to check the effect of some parameters in the spread and control of corruption. The 


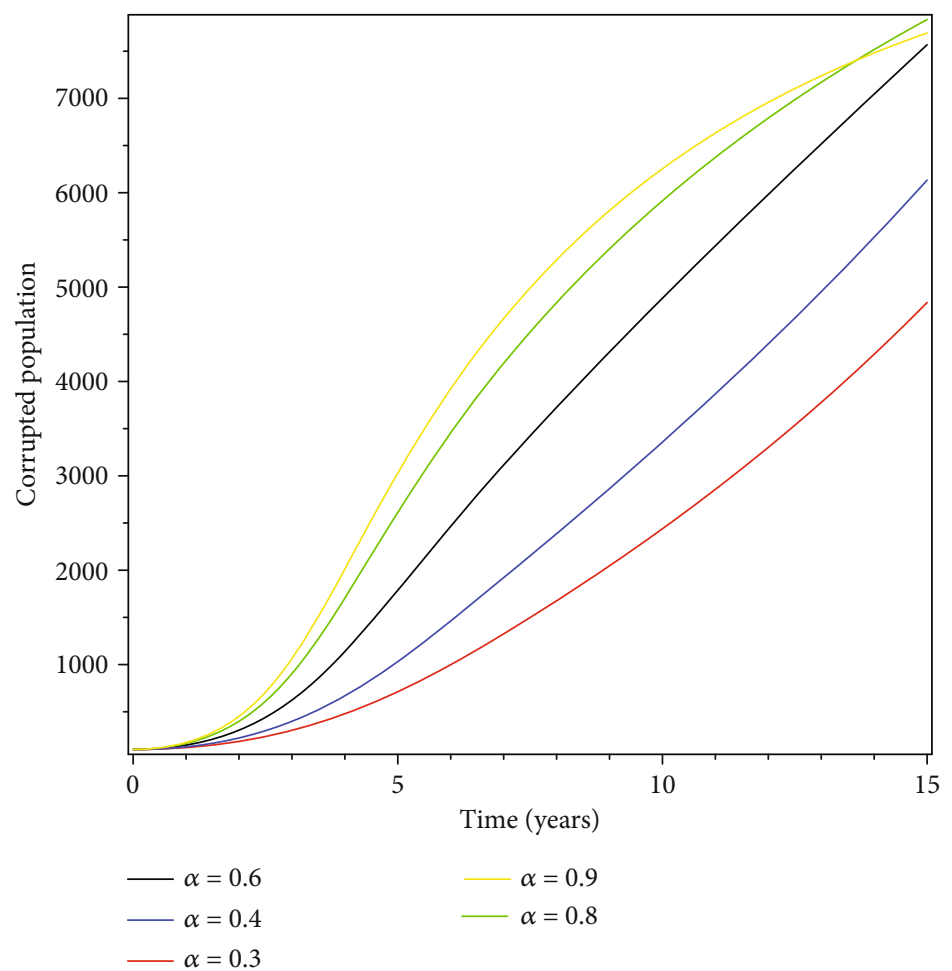

(a)

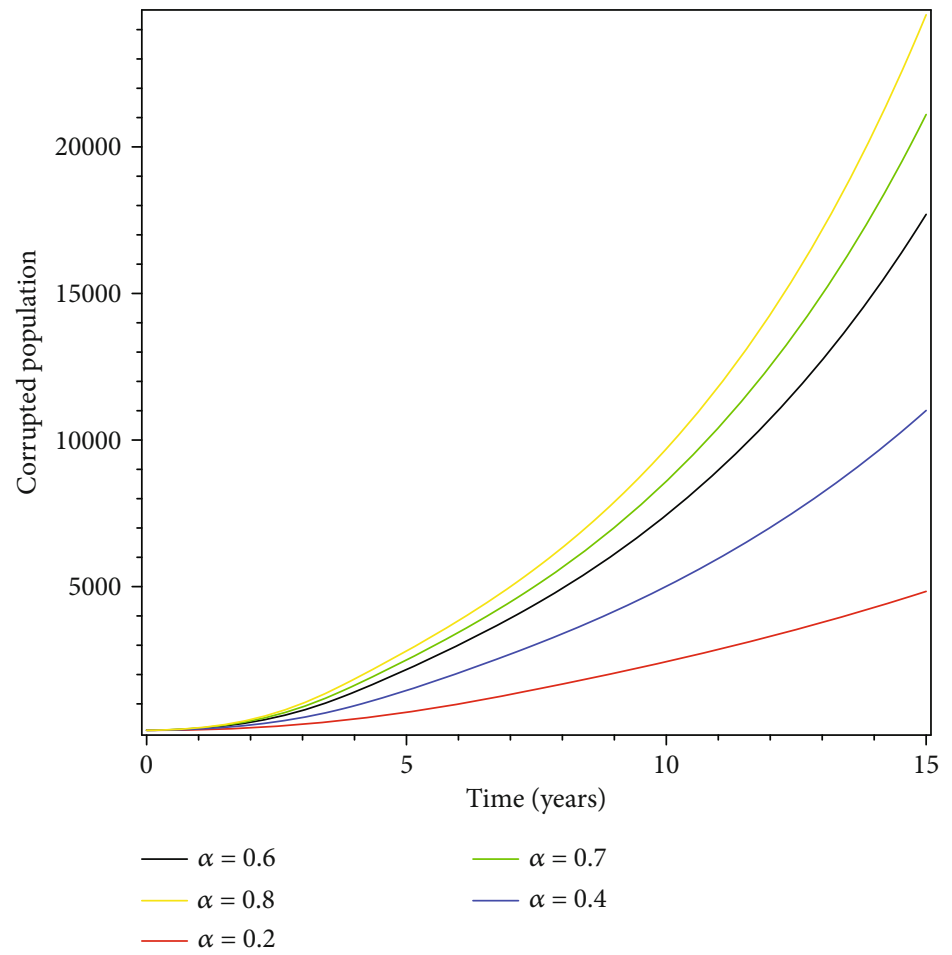

(b)

Figure 2: Effect of $\alpha$ and $\delta$ on the corrupted population.

parameter values in Table 3 and the initial conditions $S(0)=$ $10000, E(0)=0, C(0)=100, R(0)=0, H(0)=100$ are used.

5.1. Effect of $\alpha$ and $\delta$ on the Corrupted Population. As we see in Figure 2(a), we experimented the effect of changing $\alpha$ on the number of corrupted population by keeping the other parameters constant. It can be seen from Figure 2(a) that, as the $\alpha$ value increases, the number of corrupted population increases. Similarly, in Figure 2(b), we investigated the effect of changing $\delta$ on the number of corrupted population by 


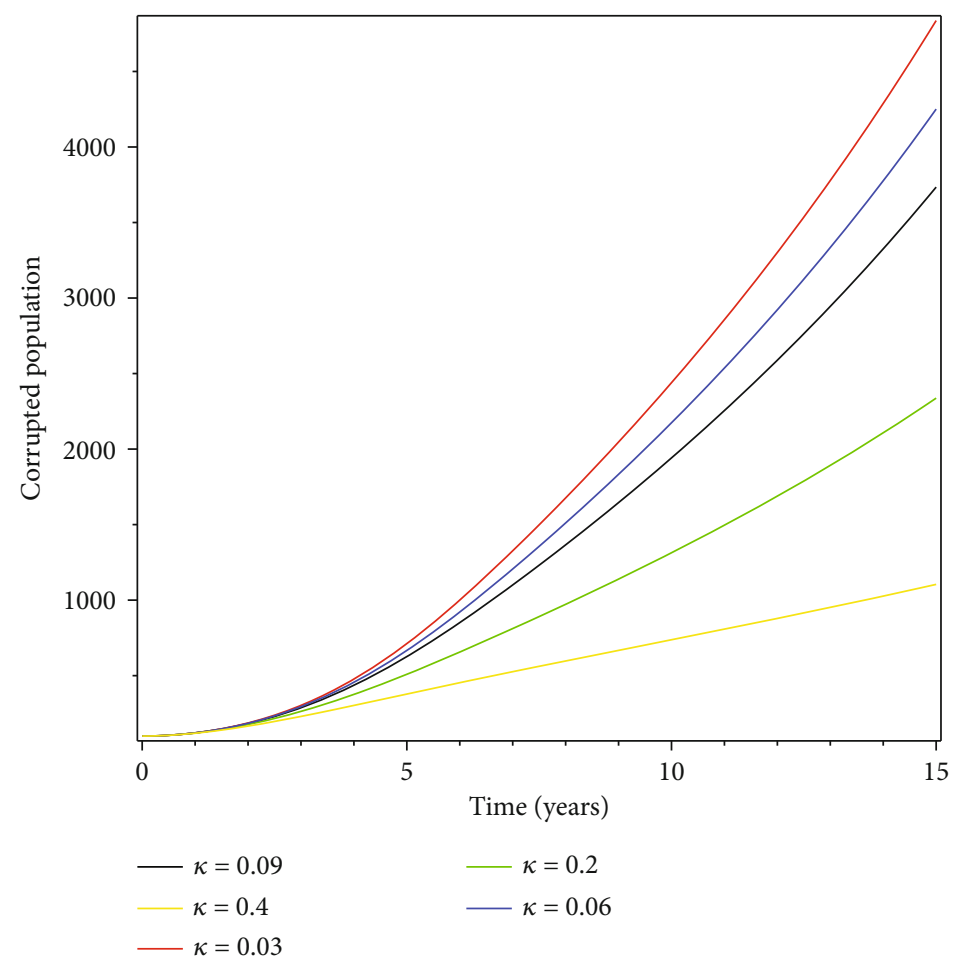

(a)

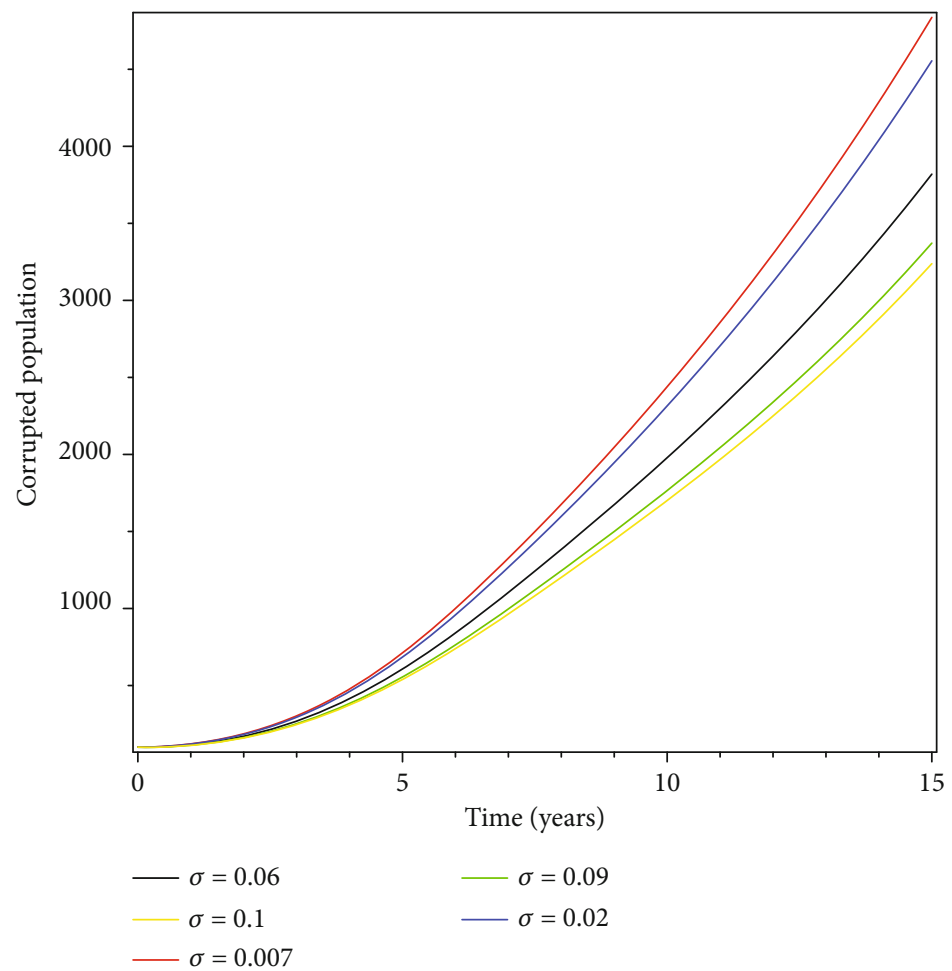

(b)

FIGURE 3: Effect of $\kappa$ and $\sigma$ on the corrupted population.

keeping the other parameters constant. It is evident from Figure 2(b) that, when the value of $\delta$ increases, the number of corrupted population also increases. Therefore, public policy makers must concentrate on minimizing the contact rate of corrupted individuals to susceptible population and reduce the transition of exposed individuals to the corrupted population.

5.2. Effect of $\kappa$ and $\sigma$ on the Corrupted Population. In Figure $3(\mathrm{a})$, we can see that $\kappa$ plays an important role in 


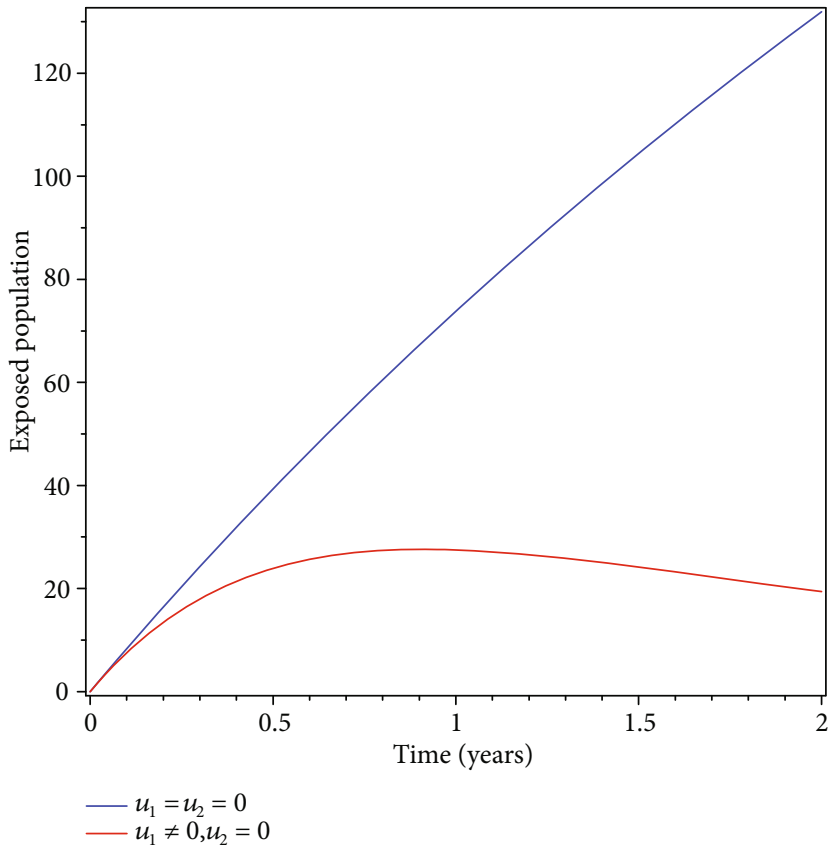

(a)

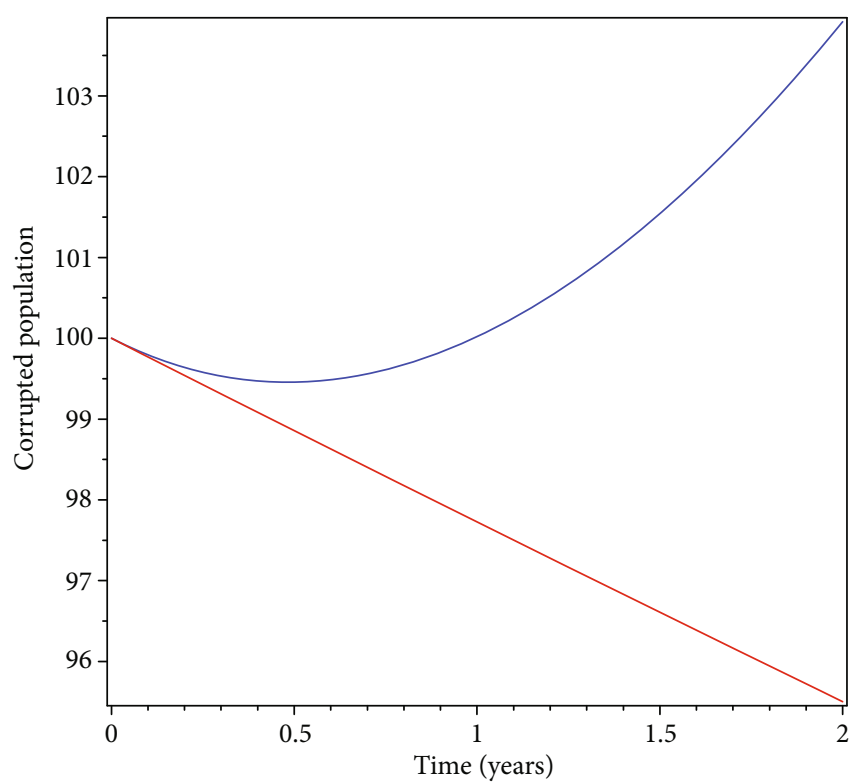

(b)

Figure 4: Simulations of the corruption model showing the effect of the optimal strategies $u_{1} \neq 0$.

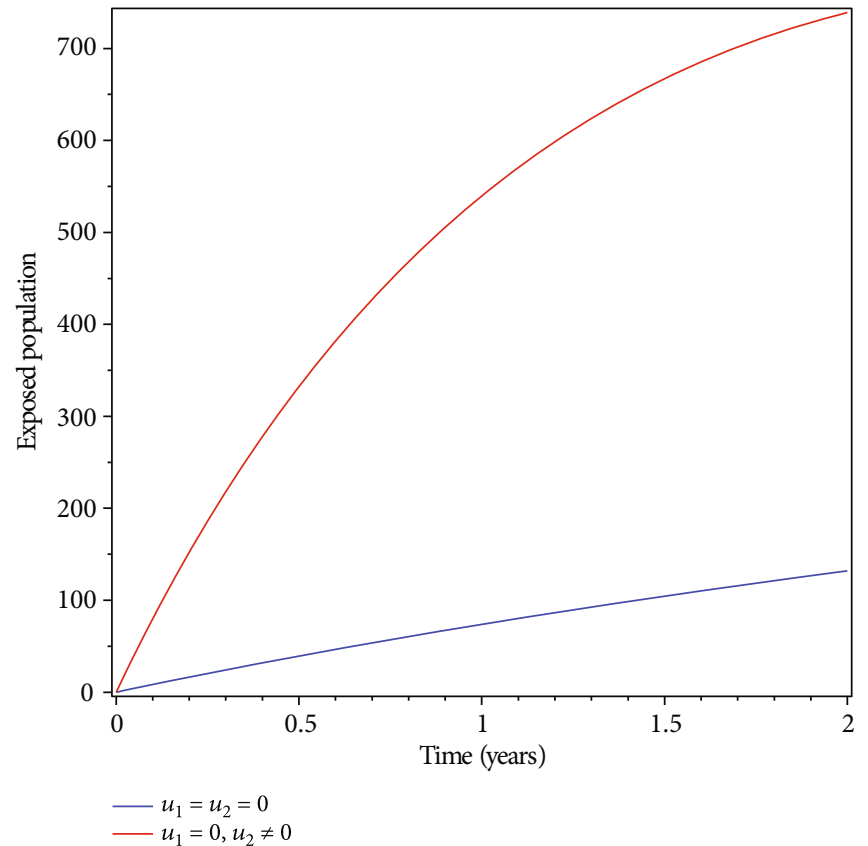

(a)

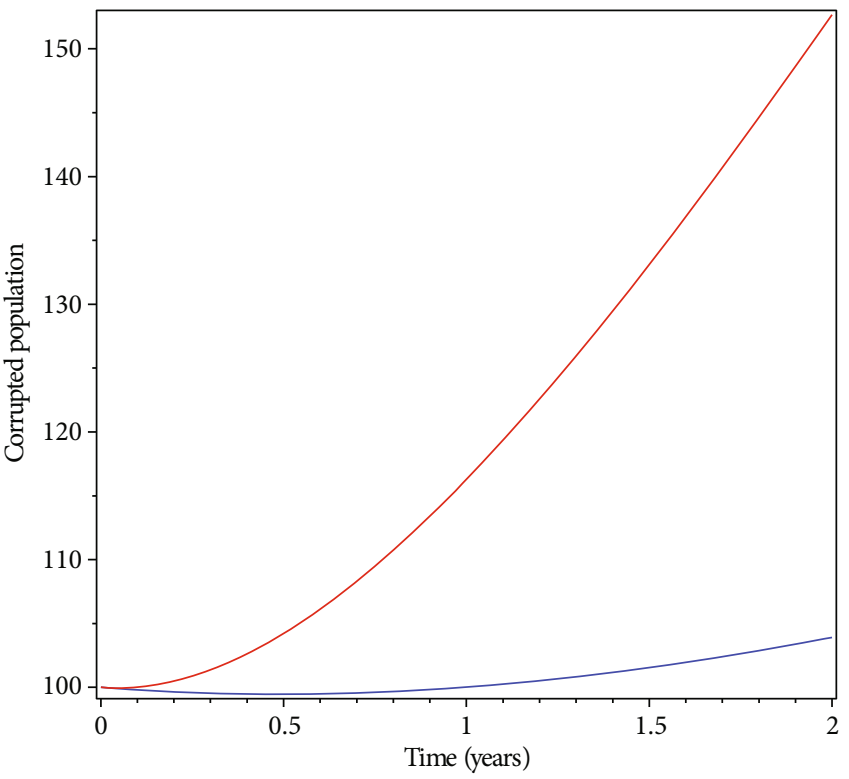

(b)

Figure 5: Simulations of the corruption model showing the effect of the optimal strategies $u_{2} \neq 0$.

reducing the number of corrupted population. When the rate at which individuals join the honest subpopulation from susceptible class increases from 0.03 to 0.4 , the number of corrupted population decreases. From Figure 3(b), the rate at which corrupted individuals join the recovered population through education and/or jail sentence increases from 0.007 to 0.1 ; the number of corrupted population decreases. Therefore, public policy makers must concentrate on maximizing the number of populations that join the honest subpopulation and corrupted population to recover from doing it.

To simulate the optimality system in (50), iterative technique is applied which gives the optimal solution. Forward fourth-order Runge-Kutta method is used to solve the state system, and backward fourth-order Runge-Kutta method is used to solve the adjoint system by considering the initial condition of the state system and the final condition of the 


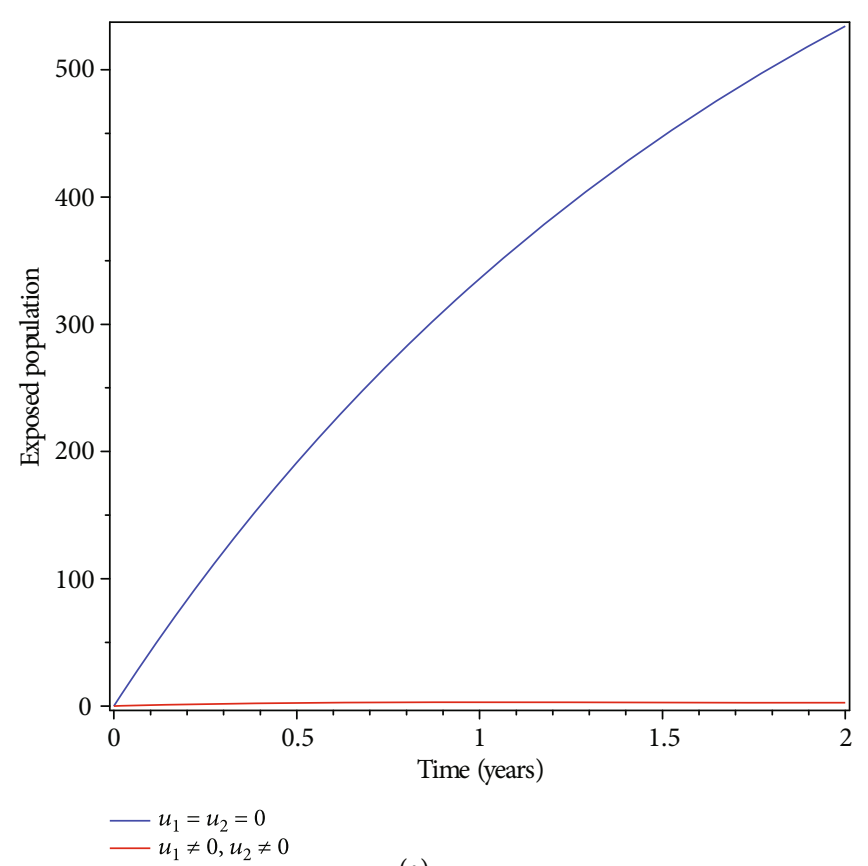

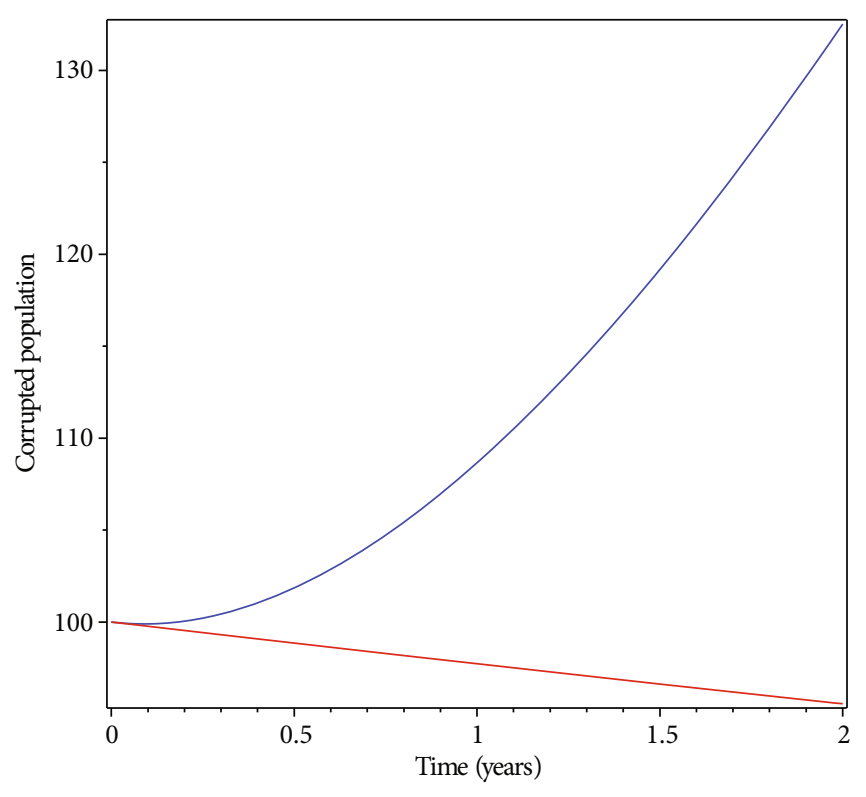

(b)

Figure 6: Simulations of the corruption model showing the effect of the optimal strategies $u_{1} \neq 0$ and $u_{2} \neq 0$.

adjoint systems. The controls continue to be updated by combining from the previous result of the controls with the characterization. The solution of the state and adjoint system is repeated by the updated controls. This condition continues repeatedly until consecutive iteration is close enough each other [23]. We proposed the following three strategies for numerical simulation of the corruption model:

(i) Strategy A: campaigning through media and advertisement about corruption $\left(u_{1} \neq 0\right.$ and $\left.u_{2} \neq 0\right)$

(ii) Strategy B: exposing the corrupted individuals to jail and giving punishment $\left(u_{1} \neq 0\right.$ and $\left.u_{2} \neq 0\right)$

(iii) Strategy C: combination of both strategies A and B $\left(u_{1} \neq 0\right.$ and $\left.u_{2} \neq 0\right)$

5.3. Strategy A: Campaigning through Media and Advertisement about Corruption. In this strategy, controls $u_{1}$ were used to optimize the objective function $J$ while we set control $u_{2}$ to zero. In Figure 4, we see that exposed and corrupted population significantly reduced when there is control compared to situation with no control. From this, we conclude that applying these strategy can reduce corruption along with time from the community but not possible to avoid it in a specified period of time. Hence, it is not an effective strategy to combat corruption from the population.

\subsection{Strategy B: Exposing the Corrupted Individuals to Jail and} Giving Punishment. Under this strategy, we optimize the objective function $J$ using the control $u_{2}$ while $u_{1}$ is set to zero. It is evident from Figure 5 that the number of exposed individuals cannot reduce using the strategy and the corrupted population seems eliminated in the first year but grows in number due to the increase in number of exposed individuals. Therefore, the strategy is not effective in fighting and reducing corruption in the population even though it reduces the number of corrupted individuals when compared with control from without control.

5.5. Strategy C: Combination of Both Strategies $A$ and B. Under this strategy, both the control measures $\left(u_{1}\right.$ and $\left.u_{2}\right)$ are used to optimize the objective functional $J$. The results of the applications of the strategy are shown in Figure 6. It can be seen from Figure 6 that the exposed and corrupted populations decrease as compared to no control. Therefore, the intervention strategy is effective in bringing down the exposed and corrupted population in the specified period of time.

\section{Conclusions}

In this paper, a mathematical model for the transmission dynamics of corruption in a population was formulated. The basic reproduction number $\mathfrak{R}_{0}$ was computed, and the stability of equilibrium points was investigated. Through Lyapunov's theory, the corruption-free equilibrium point is globally asymptotically stable whenever $\mathfrak{R}_{0}<1$ was proven. When $\mathfrak{R}_{0}=1$, bifurcation analysis of the model was proven and it exhibits forward bifurcation. The local stability of the unique endemic equilibrium whenever $\mathfrak{R}_{0}>1$ was demonstrated. Using the definition of normalized forward sensitivity, the sensitivity parameters were determined. It can be inferred that positive indices should be decreased while increasing negative indices to minimize/control corruption in a population. Then, the optimal control model was formulated by adding two time-dependent controls (campaigning about corruption through media and advertisement and exposing the corrupted individuals to jail and giving 
punishment). Optimal control theory was used to establish conditions under which the spread of corruption can be stopped and to examine the impact of a possible combination of these two controls on the transmission of corruption. The characterization of the optimal control was obtained by the application of the Pontryagin's maximum principle. It is evident from the results of the numerical simulations that the integrated control strategy should be taken to fight against corruption. The cost-effectiveness analysis of the integrated strategy would be considered in a future study.

\section{Data Availability}

The data supporting this deterministic model are from previous published articles and they have been duly cited in this paper.

\section{Conflicts of Interest}

The author declares that there is no conflict of interest regarding the publication of this paper.

\section{Acknowledgments}

I would like to thank University of Gondar for the support to do this research.

\section{References}

[1] S. Bahoo, I. Alon, and A. Paltrinieri, "Corruption in international business: a review and research agenda," International Business Review, vol. 29, no. article 101660, 2019.

[2] A. Cuervo-Cazurra, "Corruption in international business," Journal of World Business, vol. 51, no. 1, pp. 35-49, 2016.

[3] H. N. Pontell and G. Geis, International Handbook of WhiteCollar and Corporate Crime, Springer, New York, USA, 2007.

[4] F. Y. Egudam, F. Oguntolu, and T. Ashezua, "Understanding the dynamics of corruption using mathematical modeling approach," International Journal of Innovative Science, Engineering \& Technology, vol. 4, no. 4, pp. 2348-7968, 2017.

[5] F. Heimann and N. Z. Boswell, "The oecd convention: milestone on the road to reform," in New Perspectives on Combating Corruption, pp. 65-74, Transparency International, Washington, 1998.

[6] P. Milner-Smyth, "Consequences of corruption," HR Future, vol. 2017, no. Jul 2017, pp. 30-31, 2017.

[7] S. Abdulrahman, "Stability analysis of the transmission dynamics and control of corruption," Pacific Journal of Science and Technology, vol. 15, no. 1, pp. 99-113, 2014.

[8] L. Lemecha, "Modelling corruption dynamics and its analysis," Ethiopian Journal of Sciences and Sustainable Development, vol. 5, no. 2, pp. 13-27, 2018.

[9] S. R. Waykar, "Mathematical modelling: a comparatively mathematical study model base between corruption and development," IOSR Journal of Mathematics, vol. 6, no. 2, pp. 5462, 2013.

[10] S. R. Wayker, "Mathematical modelling: a study of corruption in the society of India," International Journal of Scientific and Engineering Research, vol. 4, no. 7, pp. 2303-2318, 2013.
[11] P. V. Nikolaev, "Corruption suppression models: the role of inspectors' moral level," Computational Mathematics and Modeling, vol. 25, no. 1, pp. 87-102, 2014.

[12] M. A. U. Khan, “The corruption prevention model," Journal of Discrete Mathematical Sciences and Cryptography, vol. 3, no. 13, pp. 173-178, 2000.

[13] S. Athithan, M. Ghosh, and X.-Z. Li, "Mathematical modeling and optimal control of corruption dynamics," Asian-European Journal of Mathematics, vol. 11, no. 6, article 1850090, 2018.

[14] O. M. Nathan and K. O. Jackob, "Stability analysis in a mathematical model of corruption in Kenya," Asian Research Journal of Mathematics, vol. 2019, pp. 1-15, 2019.

[15] P. Van den Driessche and J. Watmough, "Reproduction numbers and sub-threshold endemic equilibria for compartmental models of disease transmission," Mathematical Biosciences, vol. 180, no. 1-2, pp. 29-48, 2002.

[16] C. Castillo-Chavez and B. Song, "Dynamical models of tuberculosis and their applications," Mathematical Biosciences and Engineering, vol. 1, no. 2, pp. 361-404, 2004.

[17] S. M. Blower and H. Dowlatabadi, "Sensitivity and uncertainty analysis of complex models of disease transmission: an hiv model, as an example," International Statistical Review/Revue Internationale de Statistique, vol. 62, pp. 229-243, 1994.

[18] H. T. Alemneh, O. D. Makinde, and D. M. Theuri, "Ecoepidemiological model and analysis of MSV disease transmission dynamics in maize plant," International Journal of Mathematics and Mathematical Sciences, vol. 2019, pp. 1-14, 2019.

[19] H. T. Alemneh, O. D. Makinde, and D. M. Theuri, "Mathematical modelling of msv pathogen interaction with pest invasion on maize plant," Global Journal of Pure and Applied Mathematics, vol. 15, no. 1, pp. 55-79, 2019.

[20] H. T. Alemneh, O. D. Makinde, and D. M. Theuri, “Optimal control model and cost-effectiveness analysis of Maize streak virus pathogen interaction with pest invasion in Maize plant," Egyptian Journal of Basic and Applied Sciences, vol. 7, no. 1, pp. 180-193, 2020.

[21] L. S. Pontryagin, V. Boltyanskii, R. Gamkrelidze, and E. Mischchenko, The Mathematical Theory of Optimal Processes, Interscience, New York, The Mathematical Theory of Optimal Processes, New York, 1962.

[22] W. H. Fleming and R. W. Rishel, "Deterministic and stochastic optimal control," Applications of Mathematics, vol. 1, 1976.

[23] S. Lenhart and J. T. Workman, Optimal Control Applied to Biological Models, Chapman and Hall/CRC, London, UK, 2007. 